\title{
Spatial and Visual Reasoning in Biomedical Education: The Possible Contributions of Anatomical Learning
}

\author{
J Claudio Gutierrez* \\ University of California, Davis, USA \\ *Corresponding author: J Claudio Gutierrez, Department of Anatomy, Physiology and Cell Biology, University of California, Davis, \\ USA. \\ To Cite This Article: J Claudio Gutierrez. Spatial and Visual Reasoning in Biomedical Education: The Possible Contributions of Anatomical \\ Learning. Am J Biomed Sci \& Res. 2019 - 1(5). AJBSR.MS.ID.000545. DOI: 10.34297/AJBSR.2019.01.000545
}

Received: March 06, 2019 | Published: March 08, 2019

\begin{abstract}
Spatial visualization ability refers to the human cognitive ability to form, retrieve and manipulate mental models of spatial nature. Examples of validated tests that measure spatial ability and non-verbal reasoning ability (visual reasoning) are: Guay's visualization of views test, adapted version, mental rotations test, and Raven's advanced progressive matrices test, short form. There is currently information in the literature about how entry-level spatial and visual reasoning abilities may predict anatomy performance or may be enhanced with progression through the anatomy content in the medical curricula. It has been suggested that medical students possess higher spatial ability than science students, and show greater improvement in spatial ability scores than science students after learning gross anatomy. Gross anatomy is a discipline that requires the ability to visualize multiple cadaveric planes and allows the training of topographic orientation. This type of training may increase spatial and visual reasoning abilities in biomedical sciences education.
\end{abstract}

\section{Introduction}

Humans rely on visuospatial processes to perform everyday life activities [1]. Spatial ability refers to three-dimensional (3D) structure and position of objects when they are being manipulated, or mental transformation of object representations from different positions [1,2].

Regarding the general literature, there are some reports of correlations of factors such as music training, and long-term enhancement of spatial-temporal reasoning abilities in preschool children. In one such study, groups of children took either piano/ keyboard, singing or computer lessons. Children who took piano/ keyboard lessons had a significant improvement on spatial reasoning scores [3]. In a study by Gliga and Iulian-Flesner, the cognitive benefit of chess training (a game that could enhance spatial ability) was established in children. In this study, primary school students subjected to chess lessons improved their School performance in two very different disciplines: Math and Romanian language [4].

Regarding biomedical education, particularly the field of gross anatomy, it has been suggested that medical students possess higher spatial ability than science students, and also show greater improvement in spatial ability scores than science students after as little as one month of learning anatomy. The anatomic learning requires the ability to visualize multiple planes, and to transpose 3D information into 2-dimensional domains [5].

Examples of tests that measure spatial and visual reasoning (non-verbal reasoning) abilities

Guay's Visualization of Views Test: Adapted Version. This test measures spatial ability. The test measures the ability to correctly recognize 3D objects viewed from different positions. It includes 24 questions, is timed at 8 minutes to complete, and is a modified and validated version of The Purdue Visualization of Views Test (http://spatiallearning.org/index.php/testsainstruments) [6]. Questions present rotated images of 3-D objects suspended in a transparent cube. The student must identify the correct corner of the cube from which a virtual picture of the suspended object was taken. The picture of the suspended object is shown above the cube in each question. Incorrect answers incur a penalty of $1 / 6$ of a point, making the possible range of scores -4 to 24 . Average scores reported for this test are: 14.3 in fourth year dentistry students, and 10.6 in undergraduate students $[7,8]$.

Mental Rotations Test: This test measures spatial ability. The test requires selection of 3-D objects that are identical in shape to a reference object but shown in different rotational orientations (http://spatiallearning.org/index.php/testsainstruments). This test therefore measures ability to mentally rotate complex 
3-D shapes in order to find a match [10]. The 20-item test is administered in 2 parts timed at 3 minutes each, for a total of 6 minutes. Participants receive 1 point for each correct answer and -1 point for each incorrect answer, giving a range of possible scores from -40 to 40 . Average scores described by Vandenberg and Kuse for undergraduate students are: 19.06 for men and 13.17 for women [9].

Raven's Advanced Progressive Matrices Test, short form: This test measures non-verbal general visual reasoning ability (visual reasoning). This 12-question, 12-minute test is a sub-set of the original full-length Raven's Advanced Progressive Matrices Test, which was validated by Bors \& Stokes [10]. The test requires correct identification of the missing pattern in a complex design of patterns or diagrams, from a set of 8 choices. Students are not penalized for guessing, such that scores fall between 0 and 12 . Bors \& Stokes [10] reported an overall mean score for this test of 7.39 in university students [10].

\section{Anatomy in biomedical education and spatial/visual reasoning ability}

Anatomical learning has been suggested as a possible factor for improvement of spatial and general non-verbal reasoning abilities in medical students and academics. A study by Fernandez et al. [11] divided a group of clinical anatomists by anatomic skill level as experts, intermediates, novices, and unexposed controls. Spatial testing revealed that those ranked as either anatomy experts or intermediates had higher spatial visualization skills than did the anatomy novices. Further, the anatomy experts, intermediates and novices all tested higher than controls in terms of their speed of response for making correct spatial judgments [11].

Studies by Lufler et al. [12] found that medical students experienced significant visual spatial benefits during participation in the medical gross anatomy courses in the medical program [12]. Similarly, dental education appears to enhance performance of spatial ability tasks that are specific to dentistry, suggesting that it leads to the development of spatial mental models of relevant anatomy [8]. Rochford et al. [13] reported that 2nd year medical students who performed poorly on a group of spatial ability tests, also scored significantly lower on practical anatomy examinations [13]. A study by Lufler et al. [12] proposed mental rotation tests to identify students with low spatial ability and provide extra resources and tutoring for their success in anatomy [12].

The use of the Purdue visualization of rotations test has revealed interesting findings in the veterinary medical field. Provo et al. [14] made use of this test and found a significant correlation between spatial ability scores and 1st-year veterinary student performance on canine anatomy examinations. These authors suggested that students with low spatial ability may be at increased academic risk in anatomy [14]. Studies by Gutierrez et al. [15] revealed that first-year veterinary medical students showed significant increases in spatial/visual reasoning abilities. The authors concluded that veterinary medical students exposed to an integrated curriculum for 32 weeks improved their spatial visualization ability as measured by mental rotation and Guay's visualization of views tests. The same students improved their visual reasoning ability as measured by the Raven's advanced progressive matrices test, short form [15]. In a different report, Gutierrez et al. [16] described a dispersion and inconsistency of significant positive correlation between anatomy grade and spatial and visual reasoning scores suggesting these abilities may not correlate with grade or are overcome with progression through the anatomy courses. In the same report the authors found a significant difference by gender in favor of males in entry-level spatial ability scores but not for non-verbal general reasoning scores [16]. Regarding gender, Vorstenbosh et al. [5] reported a significant difference between male and female medical students in spatial ability tests. However, the males did not improve in testing with time, and, when males were excluded from the analysis, the female-only group scores significantly improved [5]. Similar differences by sex have been reported in non-medical students [17]. Studies by Provo et al. [14] revealed that male veterinary students initially had higher spatial skill scores than did females, however spatial skills were equal after 8 months of the study [14].

Some researchers are inclined to believe that androgen hormonal changes in puberty or, even before puberty, may be responsible for the gender differences in test performance. However, other researchers believe the changes emerge prior to adolescence $[18,19]$. Studies by Grimshaw et al. [20] examined the relationship between prenatal testosterone and spatial play experiences and mental rotation performance in children. The authors support the hypotheses that testosterone may act in the fetal brain to influence the development of spatial ability [20].

\section{Conclusion}

Gross anatomy is a discipline that requires the ability to visualize multiple cadaveric planes and allows the training of topographic orientation. This type of training may increase spatial and general non-verbal reasoning abilities in biomedical sciences education.

\section{References}

1. Garg AX, Norman G, Sperotable L (2001) How medical students learn spatial anatomy. Lancet 357(9253): 363-364.

2. Lohman D (1979) Spatial ability: A review and reanalysis of the correlational literature (Technical report number 8). Aptitudes research project Stanford, School of education, Stanford University, CA, USA.

3. Rauscher FH, Shaw GL, Levine LJ, Wright EL, Dennis WR, et al. (1997) Music training causes long-term enhancement of preschool children's spatial-temporal reasoning. Neurol Res 19(1): 2-8.

4. Gliga F, Iulian Flesner P (2014) Cognitive benefits of chess training in novice children. Procedia Soc Behav Sci 116(2014): 962-967.

5. Vorstenbosch MA, Klaassen TP, Donders AR, Kooloos JG, Bolhuis SM, et al. (2013) Learning anatomy enhances spatial ability. Anat Sci Educ 6(4): 257-262.

6. Elliot J, Smith IM (1983). An international dictionary of spatial tests. Windsor, United Kingdom The NFER-Nelson Publishing Company, Ltd.

7. Keehner M, Hegarty M, Cohen C, Khooshabeh P, Montello DR (2008) Spatial reasoning with external visualizations: what matters is what you see, not whether you interact. Cogn Sci 32(7): 1099-1132. 
8. Hegarty M, Keehner M, Khooshabeh P, Montello D (2009) How spatial abilities enhance, and are enhanced by, dental education. Learn Individ Differ 19(1): 61-70.

9. Vandenberg SG, Kuse AR (1978) Mental rotations, a group test of threedimensional spatial visualization. Percept Mot Skills 47(2): 599-604.

10. Bors DA, Stokes TL (1998) Raven's advanced progressive matrices: Norms for first-year university students and the development of a short form. Educ Psychol Meas 58(3): 382-98.

11. Fernandez R, Dror IE, Smith C (2011) Spatial abilities of expert clinical anatomists: comparison of abilities between novices, intermediates, and experts in anatomy. Anat Sci Educ 4(1): 1-8.

12. Lufler RS, Zumwalt AC, Romney CA, Hoagland TM (2012) Effect of visualspatial ability on medical students' performance in a gross anatomy course. Anat Sci Educ 5(1): 3-9.

13. Rochford K (1985) Spatial learning disabilities and underachievement among university anatomy students. Med Educ 19(1): 13-26.

14. Provo JL, Lamar C, Newby T (2002) Using a cross section to train veterinary students to visualize anatomical structures in three dimentions. J Res Sci Teach 39(1): 10-34.
15. Gutierrez JC, Chigerwe M, Ilkiw JE, Youngblood P, Holladay SD, et al. (2017) Spatial and Visual Reasoning: Do These Abilities Improve in FirstYear Veterinary Medical Students Exposed to an Integrated Curriculum. J Vet Med Educ 44(4): 669-675.

16. Gutierrez JC, Holladay SD, Arzi B, Gomez M, Pollard R, et al. (2018) EntryLevel Spatial and General Non-verbal Reasoning: Can These Abilities be Used as a Predictor for Anatomy Performance in Veterinary Medical Students? Front Vet Sci 5: 226.

17. Guillot A, Champely S, Batier C, Thiriet P, Collet C (2007) Relationship between spatial abilities, mental rotation and functional anatomy learning. Adv Health Sci Educ Theory Pract 12(4): 491-507.

18. Linn MC, Petersen AC (1985) Emergence and characterization of sex differences in spatial ability: a meta-analysis. Child Dev 56(6): 14791498.

19. Newcombe N, Bandura M, Taylor D (1983) Sex differences in spatial ability and spatial activities. Sex roles 9(3): 377-386.

20. Grimshaw G, Sitarenios G, Finegan JA K (1995) Mental rotation at 7 years: Relationship with prenatal testosterone levels and spatial play experiences. Brain Cogn 29(1): 85-100. 